\title{
Measurement and calibration of the nucleus position and its cross-sectional area ratio to increase the accuracy of finite element analysis
}

Jingchi Li

Third Clinical Medical College of Nanjing University of Chinese Medicine

\section{Zhipeng Xi}

Third Clinical Medical College of Nanjing University of Chinese Medicine

\section{Xiaoyu Zhang}

Third Clinical Medical College of Nanjing University of Chinese Medicine

\section{Shenglu Sun}

Third Clinical Medical College of Nanjing University of Chinese Medicine

\section{Lin Xie ( $\nabla$ xielin6508@163.com )}

Jiangsu Province Hospital of Integration of Chinese and Western Medicine https://orcid.org/00000002-2538-347X

\section{Yang Liu}

Changzheng Hospital

\section{Research article}

Keywords: Model calibration, Finite element analysis, Cross-sectional area ratio, Relative position, Range of motions, Computational accuracy

Posted Date: October 30th, 2019

DOI: https://doi.org/10.21203/rs.2.16546/v1

License: (a) (1) This work is licensed under a Creative Commons Attribution 4.0 International License. Read Full License 


\section{Abstract}

Background: As a widely used biomechanical research method, finite element analysis (FEA) is a significant tool for investigating the pathogenesis of disc degenerative diseases and optimizing of spine surgical methods. However, the definitions of the relative nucleus position and its cross-sectional area ratio do not conform to a uniform standard, thus affecting the accuracy (ACC) of the FEA. Hence, this study aimed to determine a precise definition of the relative nucleus position and its cross-sectional area ratio to increase ACC of following FEA studies.

Methods: The lumbar relative nucleus position and its cross-sectional area ratio were measured from magnetic resonance imaging data, and then calibrated and validated via FEA. Imaging data from patients without disc degeneration were recruited. The L4-L5 nucleus and disc cross-sectional areas and the distances between the edges of the annulus and nucleus were measured; the ratios between these values were calculated as $\mathrm{P} 1$ and $\mathrm{P} 2$, respectively. The FEA model was constructed using these measured values, and the relative nucleus position was calibrated by estimating the differences in the range of motions (ROMs) between the model, wherein the ligaments, facet joints and nucleus were supressed, and an in vitro study. Then, ACC were re-estimated in the model with all non-bony structures to validate the measured and calibrated indicators.

Results: The interobserver homogeneity is acceptable, and the measured P1 and P2 values are 1.22 and $38 \%$, respectively. Furthermore, an ACC of up to $99 \%$ was attained for the model under flexion-extension conditions when the calibrated P1 value (1.62) was used, with a model validation of greater than $90 \%$ attained under all loading conditions.

Conclusion: The measured and calibrated relative nucleus position and its cross-sectional area ratio increase the ACC of the FEA model, and can therefore be used in subsequent studies.

\section{Background}

Biomechanical deterioration is a key trigger for lumbar disc degenerative diseases (DDDs) 1-5. Therefore, optimised finite element analysis (FEA), which is a widely used biomechanical research method, is an ideal approach for the accurate investigation of DDD pathogenesis and treatments6-15. A series of model validation methods have been implemented by comparing the differences in the range of motion (ROM), which is a key index for lumbar motility and stability, between the FEA results and those from widely cited in vitro studies $7,10,11,14-18$. The accuracy (ACC) of the FEA models have been improved via model calibrations that used annulus and bone structures19, 20. However, inaccurate definitions of the relative nucleus position and its cross-sectional area still negatively affect the computational ACC of FEA studies.

To clarify this issue, the anterior and posterior parts of the annulus can be approximated as two differentsized quadrangular annuli on a specific plane. The 0 point is set as the pivot point of the applied torque; $\mathrm{R} 1$ and R2 are the distances between the anterior and posterior parts perpendicular of the torque axis 
centre and edges of the nucleus, respectively; and M1 and M2 are the forward and backward torques, respectively. Material mechanics theory indicates that changes in R1 and R2 origined from different nucleus position and cross-sectional areas will induce changes in $M 1$ and $M 2$, leading to ROM variations (Fig. 1). Furthermore, this change will extend to different sections if the force on the two-dimensional plane structure is extended to three-dimensional space.

However, such methods have not been defined in a uniform manner in previous FEA studies. For example, Tang et a/ modelled the centre of the nucleus as being slightly posterior to the centre of the disc and occupying $43 \%$ of the total disc volume9. Hendrik et al modelled the centre of the nucleus as being 3.5 $\mathrm{mm}$ towards the posterior side, with a size that was approximately $44 \%$ of the total disc area20. The lumbar model of Masni et al determined that the nucleus accounted for $33 \%$ of the disc volume21, whereas this rate increased to $50 \%$ in the model of Claudia et al22. Furthermore, numerous FEA studies are available in addition to the above-mentioned studies that do not address such issues. Given that individual differences are inevitable among the discs in different models, the accurate definition of the relative nucleus position should be a ratio (such as a ratio of the anterior and posterior edges of the annulus to the relative nucleus edges) rather than an ambiguous description (e.g. 'slightly posterior') or a specific value (e.g. $3.5 \mathrm{~mm}$ ).

Herein, we present an approach to accurately define such indicators in a uniform manner and increase the accuracy of following FEA studies. We first analysed magnetic resonance imaging (MRI) data of nondegenerative discs to measure the key indicators. We then constructed a 3D lumbar model to calibrate and validate our measured values. An extensive literature search indicates that no similar studies have been published to date.

\section{Methods}

\section{MRI measurements of the indicators}

\section{Collection of MRI data}

Approval for this study was obtained from the ethics committees of Jiangsu Province Hospital on Integration of Chinese and Western Medicine. Three observers, two senior spine surgeons and a seasoned musculoskeletal radiologist reviewed the lumbar MRI (Discovery MR750 3.0T, GE Healthcare; Chicago, Illinois, USA) data in our hospital over the past three years. The L4-L5 disc was selected owing to the high incidence rate of DDD. The T2-weighted imaging in the sagittal plane was selected to assess the grades of disc degeneration based on Pfirrmann et a/23, with Grade I and II degeneration considered indicative of a normal disc and included in this study 24 . The MRI data were independently reviewed, and they were included in the analysis only when all observers confirmed that the disc was a normal disc. To avoid the influence of gender difference, only male MRI data were collected for the following FEA model was constructed based on the imaging data from a 24 years old male volunteer. The kappa statistic was used to analyse the included imaging data to ensure homogeneity in the interobserver classification. 


\section{MRI measurements}

The included MRI data have been checked using the imaging workstation. The distances from the anterior and posterior edges of the annulus to the nucleus edges are set as D1 and D2, respectively. The cross-sectional areas of the nucleus and disc are set as $A 1$ and $A 2$, respectively. The ratios between the mean values of D1 and D2 and $A 1$ and $A 2$ are set as P1 and P2, respectively. These indicators (D1, D2, A1 and A2) were measured from the MRI data. Cronbach's a reliability statistic was conducted to ensure the homogeneity of the measured values. The relative nucleus position and its cross-sectional area ratio were represented as P1 and P2, which were used in the following FEA study.

\section{Calibration and validation of the FEA model}

\section{Construction of the normal model}

The L4-L5 segment 3D model was constructed using high-resolution computed tomography imaging data. $6,8,9,12,14$ The reconstructed bone structures served as the template, which was layered along the long axis. Standard curves were used to fit its contours, with the external contour of the new model overlapping the template to the maximum extent. The constructed non-bony structures were also fitted using standard curves, and the facet joint gap was set as $0.5 \mathrm{~mm} 6,9,13$. The centriod of the outlines of annulus and the inferior surface of $L 4$ were defined as a same point for the accurate placement of annulus and following calibration of annulus average radius. Six different ligaments and a capsule of facet joints were constructed during the FEA preprocessing phase11, 12, 18, 21, 25, 26(Fig. 2). The definition of the relative nucleus position and its cross-sectional area ratio were confirmed according to $\mathrm{P} 1$ and $\mathrm{P} 2$, and the outer contour of the nucleus was obtained using the same ratio reduction of the disc contour to ensure that the intervertebral disc and nucleus have the same central point for easy adjustment during the calibration process.

\section{Boundary and loading conditions}

Tetrahedral and hexahedral elements with different sizes were selected during the mesh generation, with smaller sizes and mesh refinement being used in areas that experienced serious mesh distortion. The contact type of each surface, excluding facet cartilage, was set as bounded, and the cartilage-cartilage contact was defined as frictionless $8,9,14,18,2631$. Six degrees of freedom were rigidly fixed under the inferior of $L 5$, and moments were applied to the superior of $L 46,12,16,21$. The following equation was applied to mimic the compressive load on the superior of L1 that was transferred from the superior of L4.

$F_{\text {comp }}=\operatorname{Cos}\left(\sum \theta_{i}\right) * F_{N}$

\section{Model calibration}


The calibration process was accomplished by adjusting the value of P1 via which is relatively easy adjustment in the model construction process, then estimating the ROMs under a moment of $10 \mathrm{Nm}$ and flexion-extension conditions, and finally by comparing our FEA model results from a widely cited in vitro study 19,20 .The nucleus, ligaments and facet joints were supressed during this process. Figure 3 and Table 1 show the calibration algorithm and list the material properties, respectively.

\section{Model validation}

The model was validated by evaluating the difference between ROM results from the current FEA model (with nucleus, ligaments and facet joints) and those from the in vitro study to determine if the two measured and calibrated factors reflected real biomechanical indicators under different loading conditions and determine whether the calibrated FEA model can be used in subsequent studies 27.

\section{Results}

\section{Descriptive statistics and homogeneity test}

MRI data from 43 subjects (average age of $25.6 \pm 4.3$ years) were included in the current study. The kappa values for the interobserver disc degenerative classification were in the 0.67-0.77 range, and the Cronbach's $\alpha$ values for D1, D2, A1 and A2, all of which were measured by different observers, were greater than 0.95 . These values indicated an acceptable interobserver homogeneity, with the P1 and P2 values then calculated from the measured values and used in the FEA model (Table 2).

\section{Calibration of the relative nucleus position}

The definition of ACC is shown in Figure 3, with the sensitivity of the data adjustment tested prior to calibration. We found that the ACC under flexion-extension was up to $99 \%$ when the P1 value was calibrated to 1.62. This value was confirmed and used during the model validation process (Table 3 and Figure 4).

\section{Model validation}

The validated model was modified using the calibrated relative nucleus position. We found that the ACC was higher than $90 \%$ under all of loading conditions including flexionextension, left-right lateral bending and left-right axial rotation, and was relatively higher under the flexion-extension condition (Table 4 and Figure 5).

\section{Discussion}


We explored several methods that defined the relative nucleus position and its cross-sectional area ratio, but there were still several issues with regard to our FEA models9, 20, 21, 27, 28. The data from previous studies could not be directly used in our model construction owing to individual differences and the lack of uniform standards. For example, the previously published nucleus location, wherein its centre was located $3.5 \mathrm{~mm}$ towards the posterior of the disc 20, yielded a D2 value of less than $2 \mathrm{~mm}$, which is obviously less than the minimum value measured in our study. Furthermore, the application of this value to the models constructed using imaging data from short volunteers may result in an impossible situation wherein part of nucleus lies outside the disc's boundary. The lack of uniform definition methods also leads to repeated attempts at model construction and validation. Finally, we can only define the relative nucleus position as being 'slightly posterior' to the centre of the disc, such that the lowest ACC of our model was lower than $70 \%$ and which needs to be improved furtherly, even though the model validation process was verified in our published studies. Therefore, the calibration of the relative nucleus position and the investigation of a reliable ratio to define the above indicators are vital for improving ACC of FEA, a widely used research method in the investigation of pathogenesis of DDD and optimization of spine surgical methods $6,9,13,25,28$.

The reduced T2 signal in the MRI data is closely related to disc degeneration and is generally selected to measure such a pathological change23, 29. The homogeneity test is important for ensuring the ACC and credibility of the study as it is based on subjective observer measurements 23,30 . While the kappa values between observer 1 and 3 are excellent $(>0.75)$, the rest are only acceptable $(>0.6)$. Such a phenomenon may be attributed to the small sample size and strict inclusion criteria of this study. These two constraints highlight that the slight interobserver differences can lead to obvious variations in the kappa values. Furthermore, although there is a certain degree of difference between the measured and calibrated relative nucleus positions, the modelled ROMs that are constructed from the measured nucleus position are still quite similar to the values from the in vitro study, with excellent ACC values attained (94.97\% under flexion and $96.24 \%$ under extension, Figure 4 ). The model constructed from the measured values also simulates real biomechanical indicators, such that the model calibration process further improves the ACC values based on the MRI measurements.

Notably, the nucleus itself was supressed during the model calibration process in the current study, even though the measured and calibrated values were closely associated with the nucleus. This is because R1 and $\mathrm{R} 2$ have an important impact on both $\mathrm{M} 1$ and $\mathrm{M} 2$ and their resultant ROMs. The variation in the ROMs is more likely due to the change in the approximately quadrangular annulus areas caused by the change of relative nucleus position and its cross-sectional area rather than the nucleus itself. The nucleus, ligaments and facet joints were therefore suppressed during the model calibration to investigate this factor individually. The FEA study results indicated that the retrodisplacement of the nucleus improved the ACC during the calibration process. Furthermore, the posterior structures overlapped in the model calibration under the extension condition. This phenomenon is not indicated by the computation error; rather, it is caused by the omitted contact types between the bone structures. Therefore, spatial positions are independently calculated when facet cartilages have been supressed (Fig. 4). 
Differences still exist between the ROMs in the current FEA study and the widely cited in vitro study27; these differences may be due to the supressed structures on the ROMs, even though the measurement and calibration of the relative nucleus position and its cross-sectional area ratio increase the ACC indeedly. This defect may also affect the ACC because the definition of the ligaments also lack a standard method. Furthermore, there are no published in vitro ROMs values that have been computed from models with the ligaments, facet joints and nucleus removed under lateral bending and axial rotation conditions; hence, we are unable to calibrate the FEA model under these loading conditions. This defect can provide a good explanation for the relatively lower ACC under lateral bending and axial rotation conditions. Nevertheless, we believe that the difference in the model validation is acceptable because the lowest ACC value considered in the current study is greater than $90 \%$ and the difference is far less than one standard deviation and than which in previously published studies $6,9,10,13,15,18,26$, 28. Therefore, the measured $\mathrm{P} 2$ and calibrated $\mathrm{P} 1$ values in our subsequent FEA studies can be used to increase ACC.

The current study still faces some limitations. The ratio measurement of nucleus position is based on the cross-sectional areas in a specific two dimentional plane rather than the three dimentional volume, such that the models do not capture differences in the lumbar lordotic angle. For example, obvious changes in the disc volume can be observed in the models with the same cross-sectional area ratio and different lordotic angles. Furthermore, the definition of the ligaments was accomplished based on our observer measurements and do not conform to a uniform standard, even though ligaments play a significant role of the maintenance of lumbar stability6, 11, 31, and are a key index in the ROMs. Therefore, the definition of ligaments should be investigated and calibrated in future studies to further develop more accurate FEA models.

\section{Conclusion}

The measured and calibrated relative nucleus position $(P 1=1.62)$ and its cross-sectional area ratio $(P 2=$ $38 \%$ ) could increase the ACC of the FEA model indeedly in our study, and such values can therefore be used in subsequent studies concerning the investigation of pathogenesises of DDD and optimization of spine surgical methods.

\section{References}

1.Adams MA, Roughley PJJS: What is intervertebral disc degeneration, and what causes it? Spine 2006, 31(18):2151-2161.

2.Adams MA, Freeman BJ, Morrison HP, Nelson IW, Dolan P,. \%J Spine: Mechanical initiation of intervertebral disc degeneration. Spine 2000, 25(13):1625-1636.

3.Liang J, Dong Y, Hong ZJJoOS, Research: Risk factors for predicting symptomatic adjacent segment degeneration requiring surgery in patients after posterior lumbar fusion. Journal of Orthopaedic Surgery 
Research 2014, 9(1):97.

4.Paul P, Garton HJ, Gala VC, Hoff JT, Mcgillicuddy JE, \%J Spine: Adjacent segment disease after lumbar or lumbosacral fusion: review of the literature. Spine 2004, 29(17):1938-1944.

5.Wang H, Ma L, Yang D, Wang T, Liu S, Yang S, Ding WJM: Incidence and risk factors of adjacent segment disease following posterior decompression and instrumented fusion for degenerative lumbar disorders. Medicine 2017, 96(5):e6032.

6. Hsieh YY, Chen CH, Tsuang FY, Wu LC, Lin SC, Chiang CJJCB: Removal of fixation construct could mitigate adjacent segment stress after lumbosacral fusion: A finite element analysis. Clinical Biomechanics 2017, 43(Complete): 115.

7.Lv QB, Gao X, Pan XX, Jin HM, Lou XT, Li SM, Yan YZ, Wu CC, Lin Y, Ni WF et al: Biomechanical properties of novel transpedicular transdiscal screw fixation with interbody arthrodesis technique in lumbar spine: A finite element study. J Orthop Translat 2018, 15:50-58.

8.Más Y, Gracia L, Ibarz E, Gabarre S, Peña D, Herrera AJPO: Finite element simulation and clinical followup of lumbar spine biomechanics with dynamic fixations. Plos One 2017, 12(11):e0188328.

9.Tang S, Rebholz BJ: Does lumbar microdiscectomy affect adjacent segmental disc degeneration? A finite element study. Journal of Surgical Research 2013, 182(1):62-67.

10.Wen-Hsien C, Yi-Jie K, Shang-Chih L, Chih-Wei W, Shih-Hao C, Yeung-Jen C, Jiun-Ren HJS: Comparison among load-, ROM-, and displacement-controlled methods used in the lumbosacral nonlinear finiteelement analysis. Spine 2013, 38(5):276-285.

11.Zhu R, Niu WX, Zeng ZL, Tong JH, Zhen ZW, Zhou S, Yu Y, Cheng LMJCB: The effects of muscle weakness on degenerative spondylolisthesis: A finite element study. Clinical Biomechanics 2017, 41(1):34-38.

12.Kim HJ, Kang KT, Chun HJ, Lee CK, Chang BS, Jin SYJESJ: The influence of intrinsic disc degeneration of the adjacent segments on its stress distribution after one-level lumbar fusion. European Spine Journal 2015, 24(4):827-837.

13.Chuang WH, Lin SC, Chen SH, Wang CW, Tsai WC, Chen YJ, Hwang JRJS: Biomechanical effects of disc degeneration and hybrid fixation on the transition and adjacent lumbar segments: trade-off between junctional problem, motion preservation, and load protection. Spine 2012, 37(24):1488-1497.

14.Li J, Zhang X, Xu W, Xi Z, Xie L: Reducing the extent of facetectomy may decrease morbidity in failed back surgery syndrome. BMC Musculoskelet Disord 2019, 20(1):369.

15.Ho-Joong K, Heoung-Jae C, Hwan-Mo L, Kyoung-Tak K, Choon-Ki L, Bong-Soon C, Jin S, Yeom: The biomechanical influence of the facet joint orientation and the facet tropism in the lumbar spine. Spine 
Journal 2013, 13(10):1301-1308.

16.Dreischarf M, Zander T, Shiraziadl A, Puttlitz CM, Adam CJ, Chen CS, Goel VK, Kiapour A, Kim YH, Labus KMJJoB: Comparison of eight published static finite element models of the intact lumbar spine: predictive power of models improves when combined together. Journal of Biomechanics 2014, 47(8):1757-1766.

17.Renner SM, Natarajan RN, Patwardhan AG, Havey RM, Voronov LI, Guo BY, Andersson GBJ, An HSJJoB: Novel model to analyze the effect of a large compressive follower pre-load on range of motions in a lumbar spine. Journal of Biomechanics 2007, 40(6):1326-1332.

18.Matsukawa K, Yato Y, Imabayashi H, Hosogane N, Asazuma T, Chiba K: Biomechanical evaluation of lumbar pedicle screws in spondylolytic vertebrae: comparison of fixation strength between the traditional trajectory and a cortical bone trajectory. J Neurosurg Spine 2016, 24(6):910-915.

19.Schmidt H, Heuer F, Drumm J, Klezl Z, Claes L, Wilke HJJCB: Application of a calibration method provides more realistic results for a finite element model of a lumbar spinal segment. Clinical Biomechanics 2007, 22(4):377-384.

20.Schmidt H, Heuer F, Simon U, Kettler A, Rohlmann A, Claes L, Wilke HJJCB: Application of a new calibration method for a three-dimensional finite element model of a human lumbar annulus fibrosus. Clinical Biomechanics 2006, 21(4):337-344.

21.Akiah MA, Tanaka MJCiB, Medicine: Biomechanical investigation on the influence of the regional material degeneration of an intervertebral disc in a lower lumbar spinal unit: A finite element study. Computers in Biology Medicine 2018, 98.

22.Ottardi C, Galbusera F, Luca A, Prosdocimo L, Sasso M, Brayda-Bruno M, Villa TJME, Physics: Finite element analysis of the lumbar destabilization following pedicle subtraction osteotomy. Medical Engineering Physics 2016, 38(5):506-509.

23.Pfirrmann CW, Metzdorf A,., Zanetti M,., Hodler J,., Boos N,. \%J Spine: Magnetic resonance classification of lumbar intervertebral disc degeneration. Spine 2001, 26(17):1873-1878.

24.Qasim M, Natarajan RN, An HS, Andersson GBJJoB: Damage accumulation location under cyclic loading in the lumbar disc shifts from inner annulus lamellae to peripheral annulus with increasing disc degeneration. Journal of Biomechanics 2014, 47(1):24-31.

25.Buell TJ, Bess S, Xu M, Schwab FJ, Lafage V, Ames CP, Shaffrey Cl, Smith JS: Optimal tether configurations and preload tensioning to prevent proximal junctional kyphosis: a finite element analysis. J Neurosurg Spine 2019:1-11.

26.Kang KT, Koh YG, Son J, Jin SY, Park JH, Kim HJ: Biomechanical evaluation of pedicle screw fixation system in spinal adjacent levels using polyetheretherketone, carbon-fiber-reinforced polyetheretherketone, 
and traditional titanium as rod materials. Composites Part B Engineering 2017:S1359836817302159.

27.Panjabi MM, Oxland TR, Yamamoto I, Crisco JJ: Mechanical behavior of the human lumbar and lumbosacral spine as shown by three-dimensional load-displacement curves. J Bone Joint Surg Am 1994, 76(3):413-424.

28.Li J, Xu W, Zhang X, Xi Z, Xie L: Biomechanical role of osteoporosis affects the incidence of adjacent segment disease after percutaneous transforaminal endoscopic discectomy. Journal of Orthopaedic Surgery and Research 2019.

29.Modic MT, Masaryk TJ, Ross JS, Carter JR, \%J Radiology: Imaging of degenerative disk disease. Radiology 1988, 168(1):177-186.

30.Coste J, Judet O, Barre O, Siaud JR, Lara ACD, Paolaggi JBJJoCE: Inter- and intraobserver variability in the interpretation of computed tomography of the lumbar spine. Journal of Clinical Epidemiology 1994, 47(4):375.

31.Bermel EA, Barocas VH, Ellingson AM: The role of the facet capsular ligament in providing spinal stability. Comput Methods Biomech Biomed Engin 2018, 21(13):712-721.

\section{Declarations}

\section{Funding:}

This study was funded by the Foundation for leading talent in traditional Chinese medicine of Jiangsu province(2018 SLJ0210) and Key project of jiangsu province social development (BE2019765).

\section{Conflicts of interest:}

The authors declare that they have no Conflicts of interest.

\section{Authors' contributions:}

Lin Xie, Yang Liu and Jingchi Li contributed to the concept and design of the study. Zhipeng Xi, Shenglu Sun and Lin Xie measured the MRI data. Xiaoyu Zhang and Jingchi Li contributed to the model reconstruction. Yang Liu and Jingchi Li performed the model calibration and data analysis. Lin Xie, Yang Liu and Jingchi Li wrote and checked the manuscript. All authors read and approved the final manuscript.

\section{Availability of data and materials:}

All the data of the manuscript are presented in the paper. 


\section{Acknowledgement:}

We acknowledge MSC. Tian Tang for the collection of the MRI data.

\section{Tables}

Table. 1 Material properties in the average radius calibrating model

\begin{tabular}{|c|c|c|c|c|c|c|}
\hline & \multicolumn{2}{|c|}{$\begin{array}{l}\text { Young's modules } \\
\text { (Mpa) }\end{array}$} & $\begin{array}{l}\text { Possion } \\
\text { ratio }\end{array}$ & $\begin{array}{c}\text { Cross-sectional areas } \\
(\mathrm{mm} 2)\end{array}$ & $\begin{array}{c}\text { Element } \\
\text { types }\end{array}$ & $\begin{array}{l}\text { Element sizes } \\
(\mathrm{mm})\end{array}$ \\
\hline Cortical & \multicolumn{2}{|l|}{12000} & 0.3 & I & Tetrahedral & 2.8 \\
\hline Cancellous & \multicolumn{2}{|l|}{100} & 0.2 & 1 & Hexahedral & 3.5 \\
\hline $\begin{array}{l}\text { Posterior } \\
\text { structures }\end{array}$ & \multicolumn{2}{|l|}{3500} & 0.25 & I & Tetrahedral & 4 \\
\hline Endplates & \multicolumn{2}{|l|}{1000} & 0.4 & 1 & Tetrahedral & 1.2 \\
\hline Cartilages & \multicolumn{2}{|l|}{10} & 0.4 & I & Tetrahedral & 0.45 \\
\hline Annulus & \multicolumn{2}{|l|}{4.2} & 0.1 & I & Tetrahedral & 0.3 \\
\hline Nucleus & \multicolumn{2}{|l|}{1} & 0.499 & I & Hexahedral & 1.0 \\
\hline Capsules & $7.5(<25 \%)$ & $33(>25 \%)$ & 0.3 & 30 & Cable & 1 \\
\hline ALL & $8(<12 \%)$ & $21(>12 \%)$ & 0.3 & 60 & Cable & I \\
\hline PLL & $11(<11 \%)$ & $22(>11 \%)$ & 0.3 & 21 & Cable & I \\
\hline $\mathrm{LF}$ & $15(<6.2 \%)$ & $19(>6.2 \%)$ & 0.3 & 60 & Cable & I \\
\hline ITL & $10(<18 \%)$ & $59(>18 \%)$ & 0.3 & 10 & Cable & I \\
\hline ISL & $10(<14 \%)$ & $12(>14 \%)$ & 0.3 & 40 & Cable & 1 \\
\hline SSL & $9(<20 \%)$ & $16(<20 \%)$ & 0.3 & 30 & Cable & I \\
\hline
\end{tabular}

Abbrevation: Anterior longitudinal ligament (ALL), Posterior longitudinal ligament (PLL), Ligamentum flavum (LF), Intertransverse ligament (ITL), Interspinous ligament (ISL), Supraspinous ligament (SSL).

Table. 2 Homogeneity test of measured values

\begin{tabular}{ccccc}
\hline & Average values & Cronbach's $\alpha$ & & Kappa values \\
\hline D1 & $8.87 \pm 1.51$ & 0.97 & & \\
D2 & $7.29 \pm 1.37$ & 0.96 & $1 \& 2$ & 0.72 \\
A1 & $1761.0 \pm 206.1$ & 0.96 & $2 \& 3$ & 0.77 \\
A2 & $671.4 \pm 123.8$ & 0.98 & $1 \& 3$ & 0.67 \\
\hline
\end{tabular}


1,2 and 3 stand for three observers in this study, 1 and 2 are senior spine surgeons and 3 is the musculoskeletal radiologist.

Table. 3 Calibration of nucleus relative position

\begin{tabular}{cccc}
\hline davers study $(10 \mathrm{Nm})$ & $\mathrm{P} 1$ & calibration data $\left({ }^{\circ}\right)$ & ACC $(\%)$ \\
\hline & 1.12 & 15.49 & 92.69 \\
& 1.22 & 15.87 & 94.97 \\
Flexion & 1.32 & 16.13 & 96.53 \\
$\left(16.71^{\circ}\right)$ & 1.42 & 16.31 & 97.61 \\
& 1.52 & 16.42 & 98.26 \\
& 1.62 & 16.55 & 99.04 \\
& 1.12 & -17.12 & 94.58 \\
Extension & 1.22 & -16.85 & 96.24 \\
$\left(-16.24^{\circ}\right)$ & 1.32 & -16.51 & 98.34 \\
& 1.42 & -16.39 & 99.08 \\
& 1.52 & -16.30 & 99.63 \\
& 1.62 & -16.21 & 99.82 \\
\hline
\end{tabular}

Table. 4 Model validation in different loading conditons

\begin{tabular}{cccc}
\hline FEA study $(100 \mathrm{~N}+10 \mathrm{Nm})$ & $\mathrm{F}-\mathrm{E}\left({ }^{\circ}\right)$ & $\mathrm{L}-\mathrm{R} \mathrm{B}\left({ }^{\circ}\right)$ & $\mathrm{L}-\mathrm{R} \mathrm{A}\left(^{\circ}\right)$ \\
\hline In-vitro study & 12.66 & 10.01 & 2.19 \\
This FEA study & 12.70 & 9.46 & 2.38 \\
ACC (\%) & 99.68 & 94.51 & 91.32 \\
\hline
\end{tabular}

F-E: Flexion and extension, L-R B: Left and right lateral bending, L-R A: Left and right axial rotation

\section{Figures}




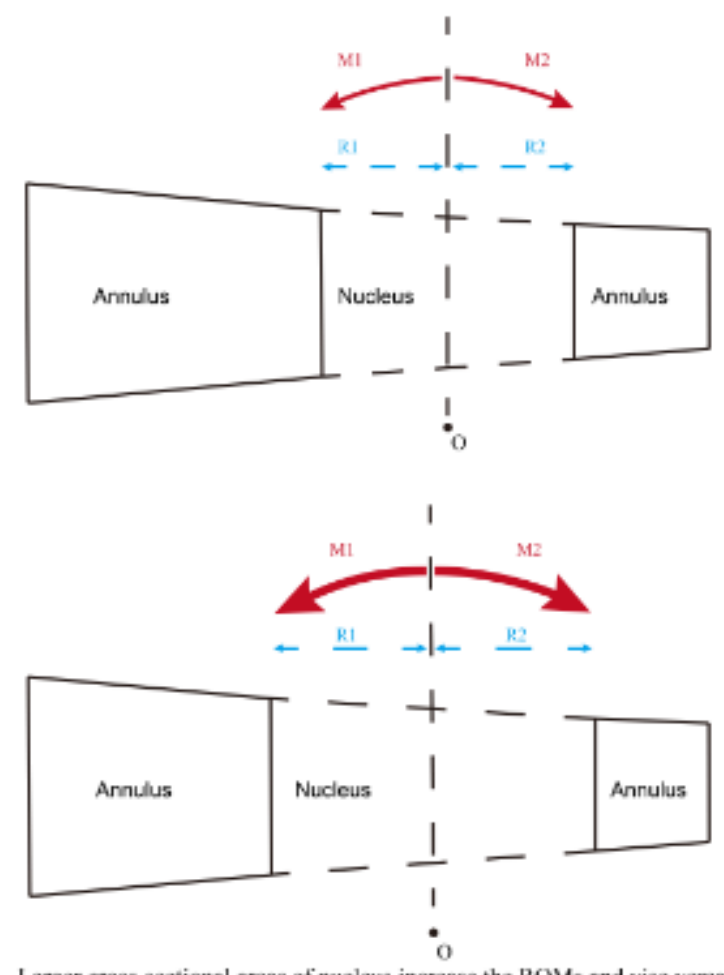

Larger cross-sectional areas of nucleus increase the ROMs and vise versa

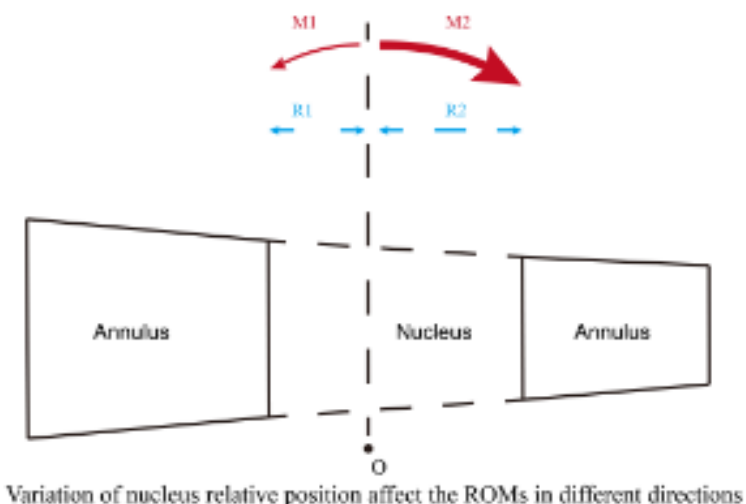

\section{Figure 1}

Effects of variations in nucleus cross-sectional areas and relative positions on the ROMs. 


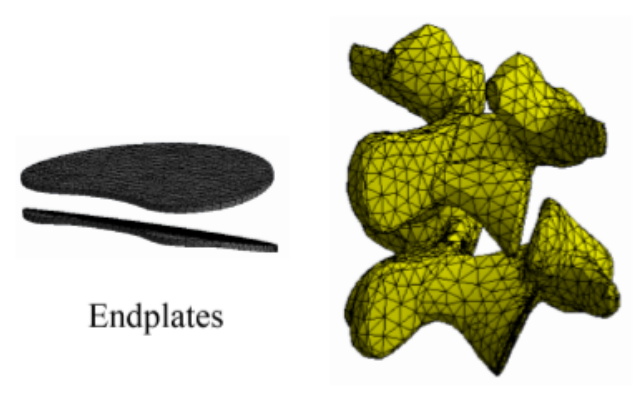

Posterior structures

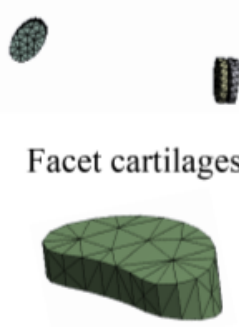

Nucleus

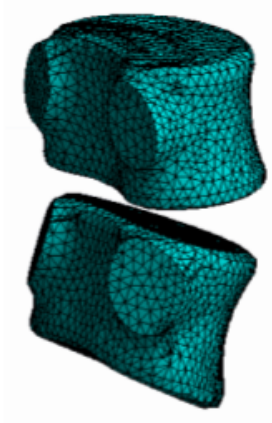

Centrum

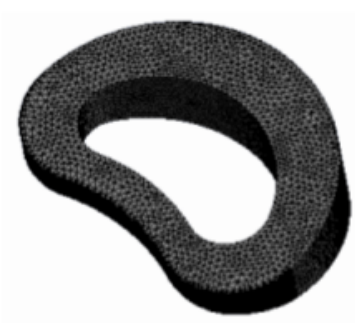

Annulus

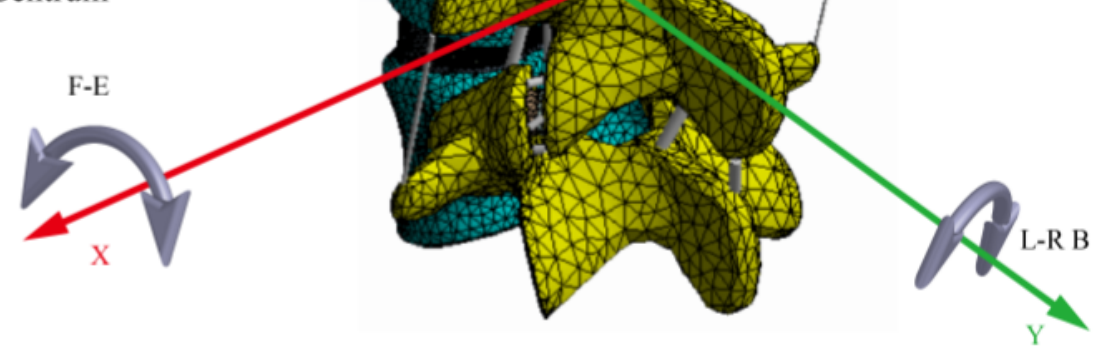

Intact model

\section{Figure 2}

Intact model and components of current models

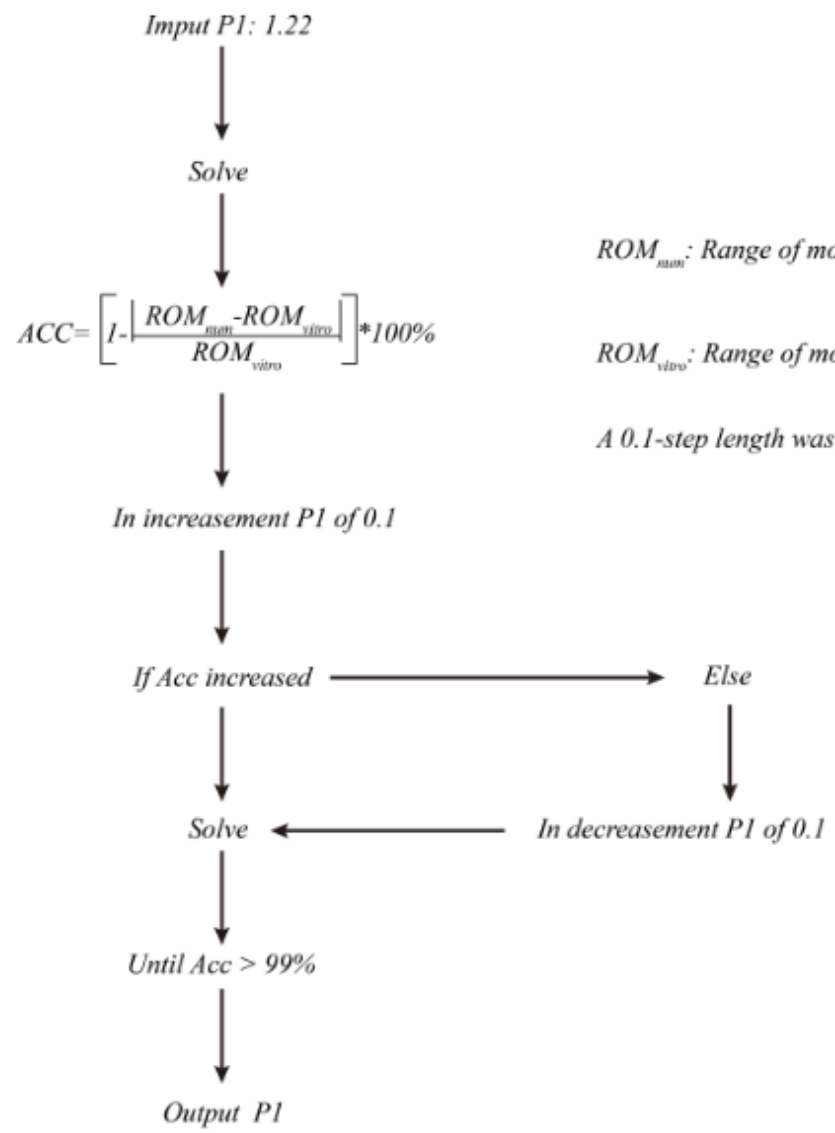


Figure 3

Calibration algorithm for the relative nucleus position.

A

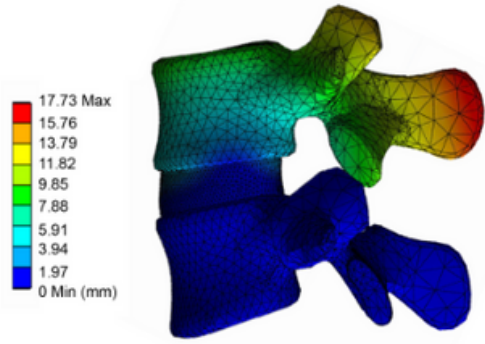

FEA ROMs $=15.87^{\circ} \quad$ ACC $=94.97 \%$

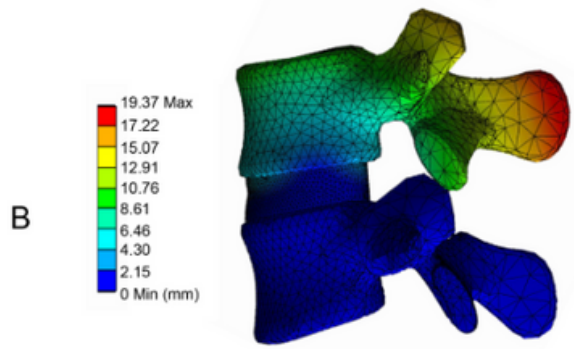

FEA ROMs $=16.55^{\circ} \quad$ ACC $=99.04 \%$

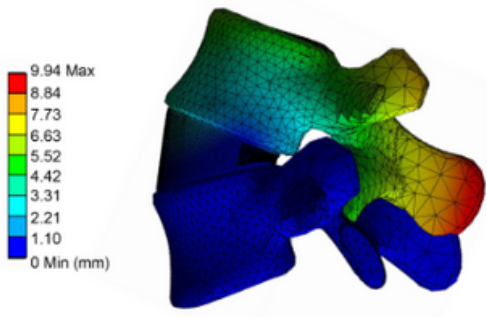

FEA ROMs $=-17.12^{\circ} \quad$ ACC $=96.24 \%$

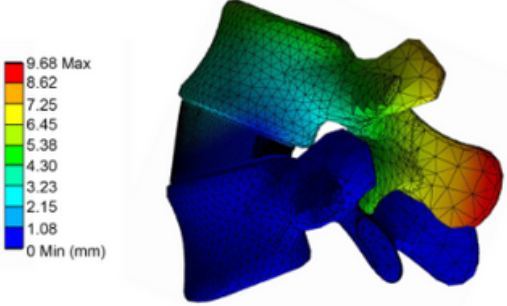

FEA ROMs $=-16.21^{\circ} \quad$ ACC $=99.82 \%$

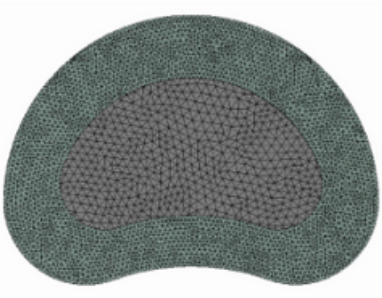

$\mathrm{P} 1=1.22, \mathrm{P} 2=38 \%$

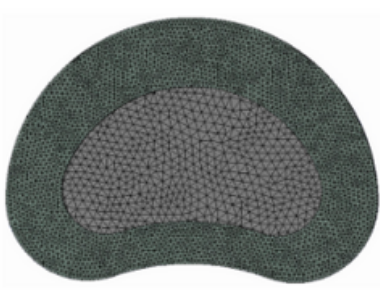

$P 1=1.62, P 2=38 \%$

\section{Figure 4}

Variations in the ROMs before and after model calibration A. ROMs before the calibration of the relative nucleus position. B. ROMs after the calibration of the relative nucleus position. 


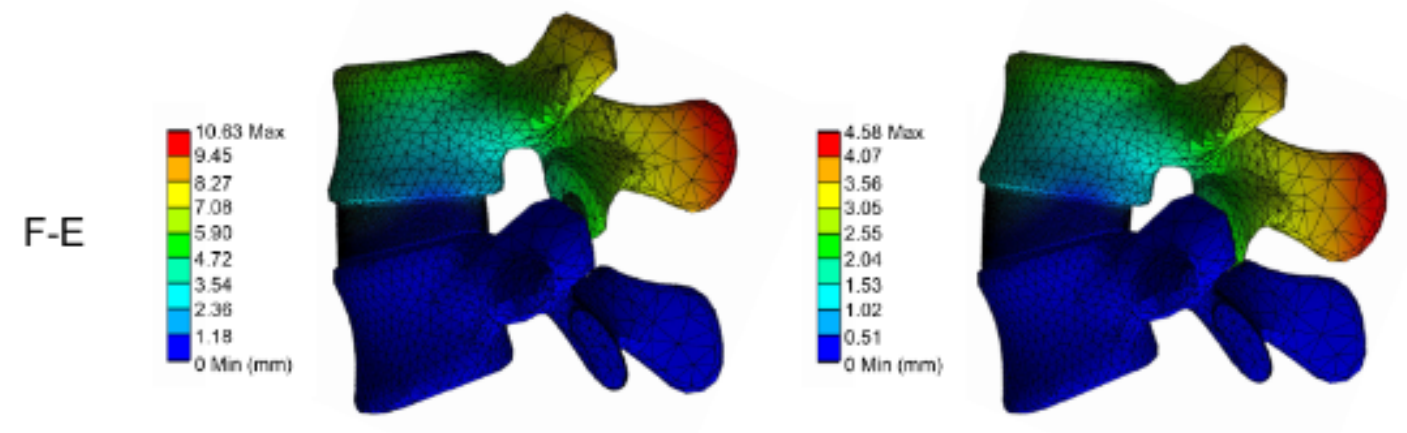

FEA ROMs $=12.70^{\circ}$ In-Vitro ROMs $=12.66^{\circ} \quad$ ACC $=99.68 \%$

L-R B
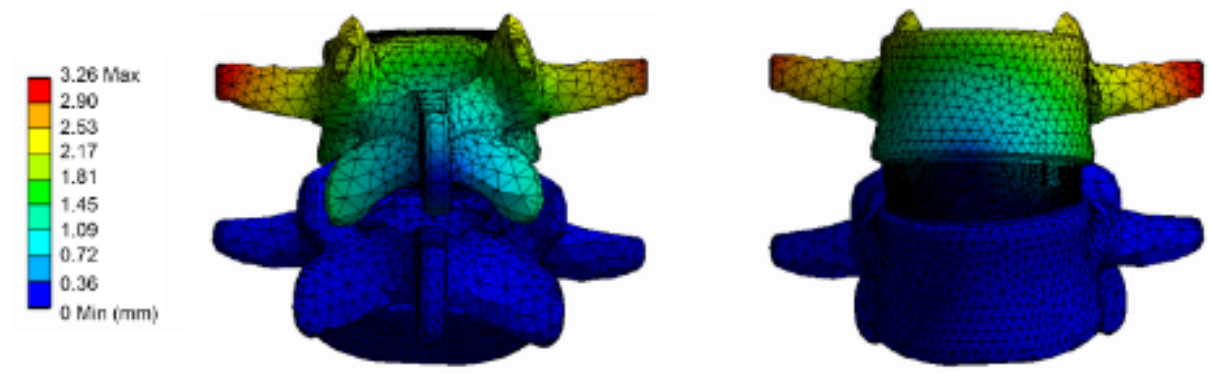

FEA ROMs $=9.46^{\circ}$ In-Vitro ROMs $=10.01^{\circ} \quad$ ACC $=94.51 \%$

L-R A
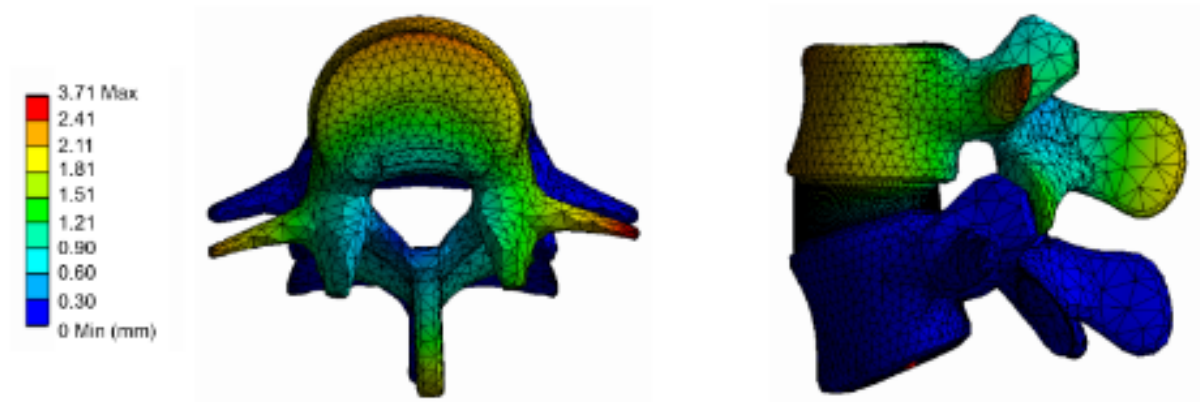

FEA ROMs $=2.38^{\circ} \mathrm{In}-$ Vitro ROMs $=2.19^{\circ}$

$\mathrm{ACC}=91.32 \%$

Figure 5

Validation of the calibrated model F-E. Flexion-Extension L-R B. Left-Right Bending L-R A. Left-Right Axial Rotation

\section{Supplementary Files}

This is a list of supplementary files associated with this preprint. Click to download.

- KeyPoints.docx 